\title{
Evaluation of Performance of Chlorinated Polyethylene Using Wireless Network and Artificial Intelligence Technology
}

\author{
Haifeng Zhang $(\mathbb{D}$ and Lianzhu Zhou \\ School of Chemical Engineering and Technology, Tianjin University, Tianjin, China \\ Correspondence should be addressed to Haifeng Zhang; zhanghaifengyc@tju.edu.cn
}

Received 31 October 2021; Accepted 27 November 2021; Published 7 January 2022

Academic Editor: Narasimhan Venkateswaran

Copyright (c) 2022 Haifeng Zhang and Lianzhu Zhou. This is an open access article distributed under the Creative Commons Attribution License, which permits unrestricted use, distribution, and reproduction in any medium, provided the original work is properly cited.

\begin{abstract}
Chemical enterprises are presently confronted with several difficult issues, including high power consumption, dangerous risk evaluation, and environmental regulation, all of which push industrial and academic institutions to develop new technologies, catalysts, and materials. Chlorinated polyethylene $(\mathrm{CPE})$ is a polymer made by replacing $\mathrm{H} 2$ molecules in high density-(C2H4) with chloride ions. CPE elastomers are made from a high density- $(\mathrm{C} 2 \mathrm{H} 4)$ backbone, and it was chlorinated using a free radical aqueous slurry technique. However, such fundamental polymer characteristics are insufficient to explain the performance characteristics of chlorinated polyethylene elastomers. Artificial intelligence (AI) has had a massive effect on all sections of the chemical sector, with tremendous potential that has revolutionized value supply chains, enhanced efficiency, and opened up new ways to the marketplace. As a result, in this research, we offer a methodology for the performance characterization of chlorinated polyethylene based on artificial intelligence (AI) and wireless network technology. The AI tools can search through enormous databases of known compounds and their attributes, leveraging the data to generate new possibilities. The dataset is first gathered. The chemical characterization is classified using the $K$-nearest neighbor (KNN) technique. This program was created to examine molecule structures and forecast the outcomes of new chemical reactions. Bayesian optimization is used to improve characterization performance. The proposed method will contribute to the future usage of AI in the chemical sector.
\end{abstract}

\section{Introduction}

Polymers are a necessary and important substance group in materials science. They are found in a wide range of products, from commonplace items like the packaging to cutting-edge technologies like lithium-ion batteries, solar cells, and 3D printing materials. There are quite a few of them. Chlorinated polyethylene (CPE or PE-C) is a soft polymer that is widely used in modern manufacturing processes. $\mathrm{PE}-\mathrm{C}$ is a high-staging, high-grade rubber that was used in conjunction with ethylene propylene, R-C4H9, $\mathrm{RCN}$, and CSM. PE-C changed with various matters. And the PE-C can be utilized frequently in the fabrication of wires and cables [1]. The PE-C and rubber matters, such as PVC profile pipes, can also be used to modify magnetic materials and ABS. Also, PE-C is created by substituting hydrogen and $\mathrm{Cl}^{-}$atoms in HDPE [high-density polyethylene]. PE-C is a white crystalline powder. It also does not have a smell or flavor. PE-C manufactured from little pressurized $(\mathrm{C} 2 \mathrm{H} 4)_{n}$ HDPE is preferable because of its stronger high-temperature thermal aging resistance [2]. PE-C has 0.93 to 0.96 (grams per centimeter cube) density, 5 to 25 million mean molecular weight, and 0.01 to 2.0 (grams per 10 minute) melt index. The chlorination level of PE-Chas notable impact on its possessions. A thermoplastic elastomer has a chlorine concentration ranging from 16 to 24 percent; an elastomer has a $\mathrm{Cl}^{-}$mixture ranging from 26 to 48 percent and so on. When the chlorine concentration is between 49 and 58 percent, the polymer has a leather-like semielastic hardness, but when it exceeds 73 percent, the polymer becomes brittle. After being subjected to a chlorine solution containing approximately 27 percent chlorine, high-pressure 
polyethylene loses its crystallinity. When a high-crystallinity low-pressure polyethylene with a chlorine content of $30 \%$ is introduced, the crystallinity is lost. The chlorine level of PEC rubber elastomer should range between 30 and $40 \%$. Chlorine is included in 25-45\% of CPE rubber. The increased $\mathrm{Cl}^{-}$mixture improves the fire blocking, permeability of air, and resistance of oil [3]. Reducing chlorine concentration, on the other hand, enlarges the cooling resistance, durability, and compact curve presentation of the PE-C. The PE-C comes as both the elastomer and resin types. $\mathrm{PE}-\mathrm{C}$ may be utilized with a combination of other materials such as PVC or other polymers, depending on the application, or maybe used solo [4]. It can also be blended with other polymers like ethylene, polypropylene, polystyrene, and even ABS. Although, a large amount of mixture volume of broad monomer atomic forms, compound chain sorting, and different polymer artificial operations presents significant challenges to analyzers due to the proper disadvantages of person logical skill when confronted with vast journals and higher-dimensional information. Because the vast majority of polymer research data is inaccessible, only a small portion of it can be utilized. Contemporary polymer research is dominated by an ineffectual "trial-anderror technique" that depends upon many tests coached by experience, significantly limiting the development of new plastic matters [5]. ML, a subset of AI, has advanced growing nowadays and is formally virtue at obtaining addition with operating vast volumes of higher-dimensional information. Many new opportunities have developed as a result of the rapid adoption of machine learning (ML) technology with its strong classification and regression capabilities, particularly in the polymer industry [6]. Nonetheless, the field of polymer informatics is still in its infancy. Researchers are attempting to improve ML algorithms and to integrate data accumulation and ML algorithms more deeply in specific applications [7]. In this paper, it is proposed using artificial intelligence (AI) in conjunction with wireless network technology to precisely assess the performance of chlorinated polyethylene in polymer research. Here, the main contributions include as follows:

(i) Improve data generation by combining higher outputcombination practical with higher output personation

(ii) The AI can then describe the synthetic chlorinated polyethylene's performance based on the generated data

(iii) Then by introducing the wireless network technology for properly storing and transmitting data securely

The remaining section of the paper can be constructed as follows. Section II provides a quick view of relevant literature. Section III explains the objective statement. Section IV contains a comprehensive explanation of the concept under consideration, while Section V examines the recommended approach. Finally, in Section VI, we conclude along with the future work.

\section{Related Works}

Several researchers focused on developing a method for analyzing the performance of several polymers including
CPE. That are all can be depicted below, Cravero et al. (2019) [8] examine the FS4RVDD algorithm's scalability and reliability. The measurement of the data bank, the modulo of the aspect subsets, noise in data, and kind of correlation were all varied and combined to create synthetic data (linear and nonlinear). In addition, the FS4RVDD results were compared to those of classic FS approaches applied to several simplified polymeric material representations. Zhao et al. (2018) [9], with a weight percentage of $\mathrm{Cl}$ of 71, a thin film $(8-10 \mathrm{~m})$ of randomly chlorinated polyethylene (CPE) is formed. CPE has a higher glass transition temperature and Young's modulus of $2.6 \mathrm{GPa}$ due to the $\mathrm{Cl}$ content, which may contribute to the material's strong electrical conductivity. The random dispersion of $\mathrm{Cl}$ molecules on the polymer chain is responsible for the well-managed medium permittivity (3-4) and lower loss at high temperatures. CPE's improved energy storing capacity and functioning temperature enable it an appealing polymeric dielectric for higher-pulse metalized film capacitor utilizations. Mondal et al. (2020) [10] show how lower percolating nanocarbon clusters reinforced polymer composite can be used to create a unique adaptable composite with substantial mechanical resilience and long-term stability. $\mathrm{PE}-\mathrm{C}$ compounds demonstrated a minimum flow threshold (13.7 weight percent) and an excellent EMI-shielding value of $42.4 \mathrm{~dB}$ when loaded with $40 \%$ weight VXC carbon black. CPE composites have been demonstrated to have excellent mechanical and EMI shielding features after thermal-air aging therapy ( $\mathrm{Li}$, et al. 2020) [11] in which EVA, chlorinated polyethylene, and rubber nitrile were combined with an electron beam before being irradiated. This was research done on the impact of $\mathrm{CPE}$ and electron radiation on the framework with its characteristics of compounds. EVA/NBR/CPE composites were studied for their mechanical characteristics, crosslinking degrees, crystallization assets, construction, and morphology. The results showed that adding $25 \mathrm{phr}$ CPE to the EVA/NBR blend system significantly increased the system's capacity while also increasing the degrees of crosslinking and the transmission temperature of glass while simultaneously decreasing the crystallization temperature and enthalpy. The mechanical assets of the compounds are enlarged firstly, and after that, they can be reduced. Michel et al. 2020 [12] here in which four spectroscopic techniquesare compared, together with ML classifiers, estimate theprecision of both user polymers and marine polymers wasteidentification, together with lessened ATR-FTIR, NIR spectroscopy,and XRF spectroscopy (MPD). These spectroscopies were all successful in identifying consumer plastic kinds using machine learning classifiers (Stefas et al. 2019). [13] uses Laser-Induced Breakdown Spectroscopy (LIBS), and distinct polymer models with similar polymer arrays with variant additions are identified and distinguished. LIBS spectroscopic data were used to classify the plastic samples using machine learning techniques like PCA and LDA. These machines learning algorithmic techniques, in particular, those combining LIBS and LIBS, offered outstanding classification results, with identification accuracies up to 100\% (Stanojevic 2021). [14] outlines the LCA air pollution cycle in waste creation and maintenance using library research. Following the completion of the investigation, a logical link was 
established between the toxicity effect and the generated $\mathrm{PCDD} / \mathrm{F}$ dioxins using Gaussian and exponential designs based on the artificial neural network technique and the tested information (Charalampous et al. 2021). [3] develops the scale correctness of produced examples using FDM procedure and assures the efficacy of the technique by developing a unique approach for selecting process parameter settings (Wang, et al. 2018). In [15], by using WNBRP mixes compatible with $\mathrm{PE}-\mathrm{C}$, liquefy compound was used to create thermoplastic elastomers (TPEs). Tensile strength and break elongation were much higher in the EVA/PE-C/WNBRP mixes than in EVA/ WNBRP ones. Furthermore, the WNBRP in the etched EVA/ $\mathrm{CPE} / \mathrm{WNBRP}$ was firmly incorporated into the EVA matrix, resulting in clean fracture surfaces. Results from the DMA showed that the Payne effect was greatly diminished when PE-C amount (5phr) in EVA/CPE/WNBRP. (Osmani and Mahmud 2021) [16] focuses on the usage of ML techniques on various polluted datasets. The classification algorithms used were FT and LSVM. Nondominated sorting genetic algorithmII is used with algorithms that help achieve an optimum solution with the minimal false-positive rate (FPR) and minimal false-negative rate (FNR) to select the perfect mix of event and nonevent information (FNR) (Xu et al. 2021). [17] estimates the polymer bandgap, and researchers used a machine learning frameworktermed as support vector regression (SVR), which used training data from DFT computation and Dragon to produce descriptors. The SVR design utilizing 16 important characteristics as inputs performed optimally for predicting polymer band gaps after feature selection with the greatest relevance and least redundancy. The SVR design's determination coefficient $\left(R^{2}\right)$ was as high as 0.824 for the leave-one-out crossvalidation and as low as 0.925 for the independent test for polymer band gaps based on DFT calculations and SVR predictions. The final product is obtained by adding $4,4^{\prime}$ methylene-bis-(2,6-di ter t-butyl phenol) (AO 4426) to an $80: 20$ mixture of chlorinated polyethylene (CPE) and polyvinyl alcohol (PVA). The damping characteristics and molecular architecture of the composites were calculated, described, and evaluated using DMTA, DSC, and FT-IR. According to the investigation, the composite PVA/CPE has only one dampening peak. However, with the addition of AO 4426, a new damping peak emerged above CPE's glass transition temperature as a result of phase separation caused by AO 4426 microcrystal aggregation under the effect of hydroxyl hydrogen bonds (Jiang and Zhang 2021). [18] gave an introduction about the emergence of the intelligent optimization algorithm, particularly the swarm intelligent optimization algorithm, which has given this scientific Endeavour fresh meaning. Currently, the field of artificial intelligence is seeing the emergence of self-creating swarm intelligence optimization algorithms that replicate species development. Intelligent algorithms are always emerging as civilization progresses and science and technology improved. Paper-plastic composite molds have been developed and put to use in the catering packaging business as a result of this. Consumption ideals have changed for the better in the modern era. People are increasingly conscious of environmental protection, and food safety is more prominently shown in their minds. A new emphasis for the adhesive-free composite film sector was created with the fast growth of the catering industry (Li et al. 2020) [19]. The author, describes the photo chlorinated $\left(20^{\circ} \mathrm{C}\right)$ and thermally chlorinated $\left(90^{\circ} \mathrm{C}\right)$ polyethylene with varying chlorine levels by weight (19\% up to $13 \%)$ that were subjected to infrared spectral spectroscopy to determine their quality. By our previous paper's microstructural highresolution nuclear-magnetic-resonance studies, 4-6 additional bands between $500 \mathrm{~cm}^{1}$ and $2000 \mathrm{~cm}^{1}$ were discovered. They spoke about how the chlorination process alters the molecular structure of the polyethylene samples they tested for chlorine (Wang et al. 2020) [20]. To create unique SPE materials, researchers advocate combining machine learning and coarse-grained molecular dynamics (CGMD). The coarsegraining of chemical species resulted in a multidimensional design space made up of physically interpretable universal descriptors. To effectively explore the space, the autonomous CGMD simulations were employed in conjunction with a Bayesian optimization (BO) technique. Researchers used this CGMD-BO approach to guide the directions to enhance the elements of the best-known electrolytes, comprising anion, secondary site, and backbone chain, which provided comprehensive explanations of the relations between lithium conductivity and intrinsic molecular material features, such as molecule size and nonbonding interaction strength (Ong 2019). [21] examines their work in the Materials Virtual Lab to design and optimize technological materials for energy storage, energy efficiency, and high-temperature alloys; (ii) build scalable quantum-accurate models; and (iii) increase the speed and accuracy of reading characterization spectra (Yamada, et al. 2019) [22]. XenonPy.MDL, a pre-trained model library, is currently being developed. Pretrained models for small molecules, polymers, and inorganic crystalline solids total more than 140 000 in this first release. Pretrained models are accompanied by several notable breakthroughs in transfer learning, such as generating models with just a few hundred material properties. They also show how strategic model transfer may improve extrapolative prediction ability. Even though transfer learning has shown nontrivial transferability across distinct features that transcend the many fields of materials science, our study has revealed underlying bridges between small molecules and polymers as well as between organic and inorganic chemistry (Kim et al. 2020) [23] in which the model's behavior should be shown to augment current thermodynamic information by learning representations of materials that match synthesisrelated features. Lastly, they use the model to test for the synthesizability of new perovskites that have been suggested (Vivanco-Benavides, et al. 2022). [24] shows that the quantity of information available from experiments is surprisingly similar to what is found when modeling uncontrolled physical characteristics of carbon nanotubes with the use of algorithms. Specifically, in the analysis of some nanostructure, the use of ANNs, irregular forests, and $\mathrm{KNN}$ is emphasized, particularly in the analysis of nanostructures. These methods are important. It has been found that carbon nanotubes have a wide range of interesting physical and chemical characteristics. For thermal, electrical, and electronic characteristics, the theory of density functional and dynamics of molecular findings still needs to be supplemented by machine learning results. Chiral-geometricalvariables were employed to analyze the 


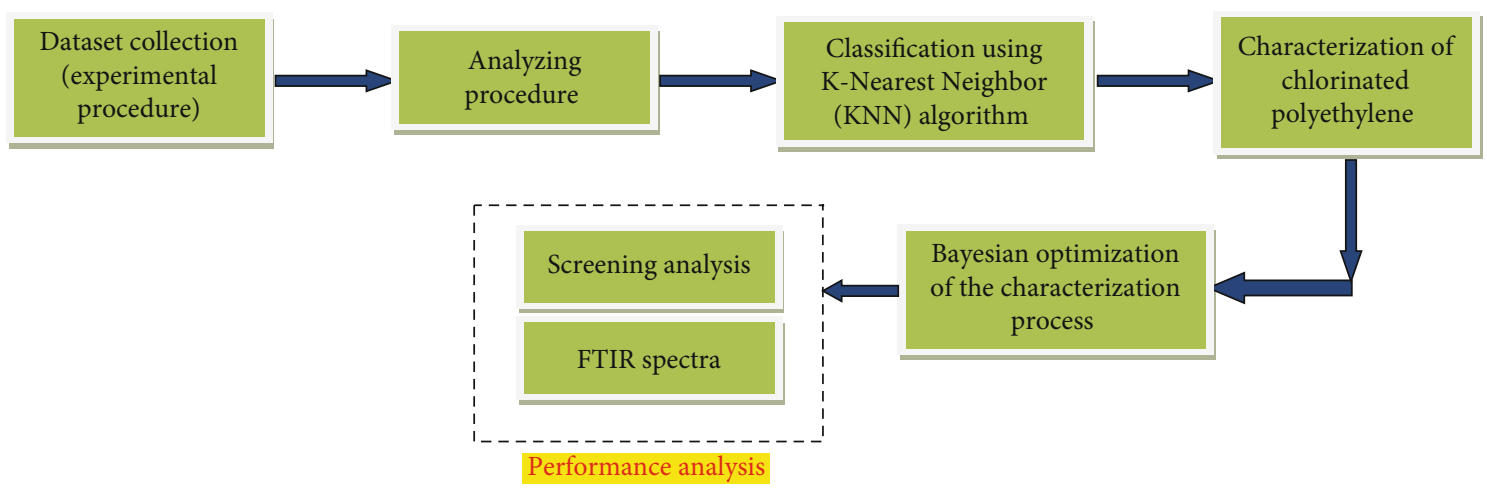

FIGURE 1: Flow of the proposed methodology.

quiver feedback of carbon nanotubes; consequently, several exact predictions were needed to forecast the mentioned waves when it comes to mechanical characteristics. To explain the thermionic and vibrational characteristics of carbon nanotubes using machine learning, a certain number of iterations must be completed (Zhao et al. 2021) [25]. JAMIP is a Python framework created by Jilin University to fulfill the research needs of computational material informatics; JAMIP is a data-driven infrastructure powered by artificial intelligence. It consists of a raw material manufacturing plant, a high-throughput firstprinciples computations engine, an automated task submission and progress monitoring system, and data extraction, management, and storage system. To aid high-throughput computations, certain elements such as an inorganic crystal structure prototype library and critical machine learning modules related to functional material investigations have been incorporated. Using halide perovskites as an example, they demonstrated how their newly developed technique may be utilized to analyze optoelectronic semiconductor materials informatics. Because it adheres to the ideas of automation, flexibility, dependability, and intelligence, the JAMIP code is a promisingly powerful instrument in the rapidly expanding field of computational materials informatics (Agrawal and Choudhary 2019). [1] describes the benefits, limitations, and current applications of deep learning to various forms of material data. Advances in deep learning, together with increased access to materials databases and big data generally, hold great potential for speeding up the discovery, design, and implementation of next-generation materials (Ünügül and Karaağaç 2021) [26]. Chlorinated polyethylene and chloroprene rubber mix silane vulcanization at low temperatures was examined. Instead of employing an extrusion technology, which is widely employed for predicted applications, rubber compounds were manufactured using an internal mixer. Rubber compounds were investigated for their rheological and dynamic characteristics. Vulcanization was followed by investigations into physic mechanical and thermal stress-relaxation characteristics as well as morphological and temperature scans. To discover the vulcanization reaction process, structural research was carried out utilizing Fourier transform infrared spectroscopy and $\mathrm{X}$-ray photoelectron spectroscopy. At $110^{\circ} \mathrm{C}$ and water, all of the compound compositions could be vulcanized. In comparison to epoxy silane, amino silane was shown to be more effective [27].

\section{Proposed Work}

This section explains the flow of the proposed method. Figure 1 shows the schematic representation of the proposed methodology.

3.1. Dataset Collection. In a sealed, agitated vessel or reactor, polyethylene was chlorinated in an aqueous slurry stage. In 8 liters of water, $1 \mathrm{~kg}$ of polyethylene was mumbled. The slurry was treated with 2 milliliters of nonylphenol surfactant (to lower surface tension) and $45 \mathrm{~g}$ of talc (to prevent agglomeration). The slurry's temperature was raised to around $95^{\circ} \mathrm{C}$, and the treatment began by injecting $1 / 2 \mathrm{l} / \mathrm{m}$ of chlorine gas while being exposed to ultraviolet illumination. Behind 900 seconds, the reactor pressurized force was enlarged to 2.8100000 pascals, and the reaction was held at this pressure for 5 minutes. After chlorination, the slurry was transported to another agitated tank to neutralize any remaining $\mathrm{HCl}$ before being cleaned and washed in an $80^{\circ} \mathrm{C}$ caustic batch. The polymer was dried at a temperature of around 40 degrees Celsius. CPE-1 was screened towards several fractions depending upon the distributed measurement. Here, chlorine levels in the various fractions were determined using the DIN EN ISO 1158 technique.

3.2. Analysis Flow. The OFC technique can be used to determine the amount of sulfur created when the required organic molecules are combusted in an oxygen flask. Such molecules could contain chlorine, bromine, iodine, fluorine, or sulfurcompounds. The base of the DINENISO1158 technique is a similar oxygenflask combustion technique.

The CPE representative model was weighed with an accuracy of $0.01 \mathrm{mg}$ and deposited on filter paper cut in the $50-70 \mathrm{mg}$ range. The paper trail was projecting from the platinum helix.

In the container, the water, potassium hydroxide solution (100 grams per liter), and hydrogen peroxide solution (300 grams per liter) are filled in the scales of 15 milliliter, 4 milliliters, and 1 milliliter, respectively. Also, via fiber (glass) tube, the oxygen can be given to replace the air at 250 milliliters per minute for 300 seconds ( $5 \mathrm{~min}$ ).

The back end of the paper was set to fire using a gas fire. The platinum wire-conveying stopper was firstly inserted, and the paper has been set ablaze. After combustion had 
Input: training samples $A$, test samples $B$

Output: Class labels of $Y$

* Training Phase *

Training the optimum-K-scoresofevery training sample

Utilizing ID3 technique to developKNN with training sample and their associatedoptimum-K-scores

Preserving the optimum-K-scores of training sample in every node

* Test Phase *

Attaining the optimum-K-scores of test sampleemploying KNN

Forecasting test labels employing conventionalkNNtechnique with trainedoptimum-K-scores on every training sample

Algorithm 1: Pseudocode of the Proposed KNN Method.

completed, the container was raised upstanding. The container has been stoppered along refrigerated below a cool water flow to enable rapid and complete occupation of the generated HCL acid.

Behind 1800 seconds, the contents of the container were moved into $250 \mathrm{ml}$ Erlenmeyer, and the container has been cleaned and transported to Erlenmeyer, yielding the last volume of around $40 \mathrm{ml}$. The Erlenmeyer flask was filled with 1 gram of $\mathrm{NaNO}_{3}$ and 2.5 milliliters of $\mathrm{HNO}_{3}$ liquid $(2 \mathrm{~N})$. The mixture was brought to a rolling boil for 5 minutes. After cooling, $1 \mathrm{~mL}$ of $50 \mathrm{~g} / 1$ potassium chromate solution was added, and the solution was measured through titration with silver nitrate solution $(0.1 \mathrm{~N})$. Equation $(1)$ is used to compute the chlorine concentration in the solution.

$$
\mathrm{Aci}=3.5455 \times \frac{0.1 \times\left(V_{1}-V_{2}\right)}{m},
$$

in which

Aci is the chlorine content of CPE.

$V_{1}$ and $V_{2}$ represent the volume of $\mathrm{AgNO}_{3}$ mixture used for the determination (in terms of milliliters) and the volume of silver nitrate solution used for the blank test, respectively (inmilliliter). $V_{2}$ is typically 0 for pure materials and stilled equipment. $m$ denotes the mass of the CPE sample (in grams).

\subsection{Classification Using K-Nearest Neighbor (KNN)} Algorithm. The key benefit of KNN over other algorithms is that it can be utilized to classify several classes. As a result, if the data has more than two labels or, to put it another way, if you need to categorize the data into more than two categories, $\mathrm{KNN}$ is a good choice.

The dataset is split into training and test samples. 50\% percentage of the solution is considered as training sample, and remaining $50 \%$ of the solution is taken for test samples. The kNN technique's methodology is predicated on the intuitive notion that samples from the same class must have similar feature spaces. As a result, for an indeterminate class test sample, we could simply calculate the distance between the test sample and all of the training samples and assign the class indicated by the test sample's kNNs. The proposed KNN technique condenses the training set from all training samples into a subset, namely, the neighbors of the test sample's nearest neighbors and all test sample's neighbors. As a result, we expect the set $S$ to nearly completely cover all of the training samples' nearest neighbors.

3.4. Bayesian Optimization (BO) of the Characterization Process. The Bayesian optimization (BO) method is well known for optimizing expensive opaque functions. It depends upon the probability model of to-be-optimized unspecified goal $f(a)$. The opaque function $f(a)$ is called indefinitely until the budget is exhausted. Queries are made up of $f$ evaluations at different hyperparameter configurations a1,..., and that is determined using an explore exploit trade-off criterion or acquisitiveness function. The hyperparameter layout that corresponds to the greatest question is then come back. Applying Gaussian process (GP) advance to $f$ and computing the posterior GP depends on the noticed questions $f(\mathrm{a} 1)$..., and $f$ is a common method (an). The posterior GP is defined by the posterior mean function and a posterior variance function, both of which must be assessed when the acquisition function is evaluated for each subsequent query off.

A prominent acquisitiveness function means an expected improvement (EI), and it can be explained as the sum of development expected in an assessment over present minimal $f\left(a_{\text {min }}\right)$. For a Gaussian predictive distribution, EI can be explained in the closed formation as eqn. (2).

$$
\begin{gathered}
E I(a)=E\left[\max \left(0, f\left(a_{\min }\right)-f(a)\right]=\sigma^{2}(a)(z(a))+\Phi_{\mathrm{n}}(\mathrm{z}(\mathrm{a}))\right), \\
z(a):=\frac{\mu(a)-f\left(a_{\min }\right)}{\sigma^{2}(a)} .
\end{gathered}
$$

In response to the need for information, other acquisition functions have emerged. Standard acquisitions are just concerned with the goal $f(a)$ and do not take into account any additional limitations. The goal of this study is to optimize an opaque function $f(a)$ that corresponds to goodness restrictions $c(x)$, with $R+$ indicating how closely the associated fairness rule should be imposed.

\section{Performance Analysis}

4.1. Progress of Reaction. The Cl-concentrations of CPE-(1-3) are roughly 6.5, 12, and 34 percent, as per DIN EN ISO 1158. In terms of response time, Figure 2 displays the reaction time of three CPE-(1-3) productions.

As can be observed, as the length of the chlorination reaction grows in this interval, the rate of the reaction 


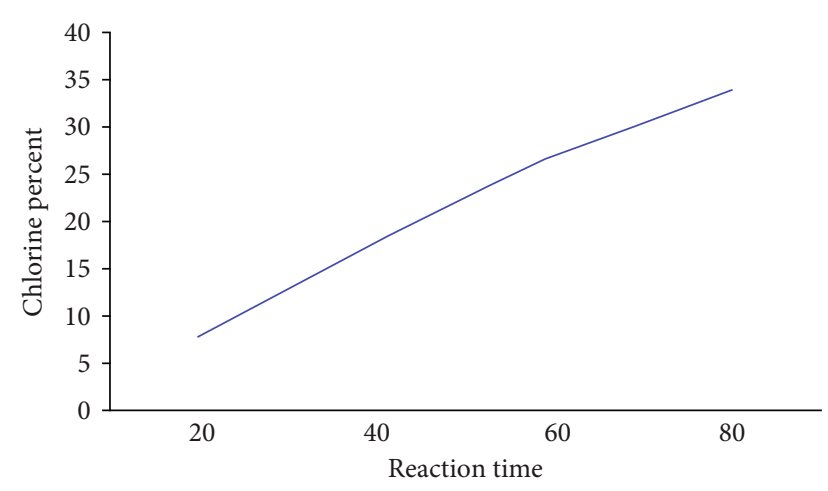

FIgURE 2: Chlorine content of products vs. time (minute).

TABLE 1: Differential screening analysis.

\begin{tabular}{lcccc}
\hline \multirow{2}{*}{ Distributed size $(\mu \mathrm{m})$} & \multicolumn{4}{c}{ Wt. of polyethylene and objects $(\mathrm{g})$} \\
& PE & CPE-1 & CPE-2 & CPE-3 \\
\hline 2000 & 104 & 110 & 115 & 136 \\
1015 & 383 & 407 & 427 & 510 \\
500 & 276 & 294 & 310 & 370 \\
300 & 90 & 96 & 101 & 121 \\
150 & 105 & 112 & 118 & 142 \\
100 & 42 & 45 & 47 & 57 \\
Total summation & 1000 & 1065 & 1119 & 1338 \\
\hline
\end{tabular}

increased gradually. This approach is beneficial and shows that the chlorination procedure was completed effectively. This graphic will be used in kinetic reaction researches in the future.

4.2. Screening Analysis. Six sieves of varied (mesh) diameters, comprising 100, 150, 300, 500, 1015, and 2000, were employed for differentiated screening analysis of the initial $\mathrm{PE}$ and created CPEs. Polyethylene, CPE-1, CPE-2, and CPE-3 were all screened and analyzed. Table 1 displays the results.

The masses of exceptionally larger or smaller molecules are lighter than the masses of average molecules, as per Table 1. PE molecules with 100 micron sizes, for instance, weigh roughly $42 \mathrm{~g}$, but those with 2000 micron diameters weigh $104 \mathrm{~g}$.

In addition, the table demonstrates that CPE-(1-3) was growing weights in contrast to physical education. As a consequence of the chlorination process, the mass of the products has increased. For instance, in the CPE 2 procedure, the overall sum of the final output from the reactors was $1119 \mathrm{~g}$ per 1000 grams $\mathrm{PE}$, with the addition of 119 grams representing the mass of $\mathrm{Cl}^{-}$introduced to the physical education chains, resulting in higher weight. Table 2 , on the other hand, expresses the amount of increased weight per created CPE compared to the starting PE for a certain particles size. The proportion of chlorine per generated CPEs of various sizes is shown in Table 3 .

4.3. Fourier Transfer Infrared (FTIR) Spectra. These materials have been produced as a powder with a diameter of
TABLE 2: Increase in chlorine content.

\begin{tabular}{lccc}
\hline $\begin{array}{l}\text { Size distribution } \\
(\mu \mathrm{m})\end{array}$ & \multicolumn{3}{c}{$\begin{array}{r}\text { The difference in weight between objects } \\
\text { and polyethylene }(\mathrm{g})\end{array}$} \\
\hline 2000 & $m_{\mathrm{CPE}-1}-\mathrm{m}_{\mathrm{PE}}$ & $m_{\mathrm{CPE}-2}-m_{\mathrm{PE}}$ & $m_{\mathrm{CPE}-3}-m_{\mathrm{PE}}$ \\
1015 & 6 & 11 & 32 \\
500 & 24 & 44 & 127 \\
300 & 18 & 34 & 94 \\
150 & 6 & 11 & 31 \\
100 & 7 & 13 & 37 \\
\hline
\end{tabular}

TABle 3: Chlorine percentage based on size dispersion.

\begin{tabular}{lccc}
\hline \multirow{2}{*}{ Distributed size $(\mu \mathrm{m})$} & \multicolumn{3}{c}{ Percentage of chlorine for various } \\
particle sizes & \\
& CPE-1 & CPE-2 & CPE-3 \\
\hline 2000 & 5.7 & 10.5 & 31.2 \\
1015 & 6.2 & 11.6 & 33.1 \\
500 & 6.5 & 12.3 & 34.0 \\
300 & 6.6 & 12.2 & 35 \\
150 & 7.1 & 12.3 & 35.7 \\
100 & 8.3 & 13.1 & 36.9 \\
\hline
\end{tabular}

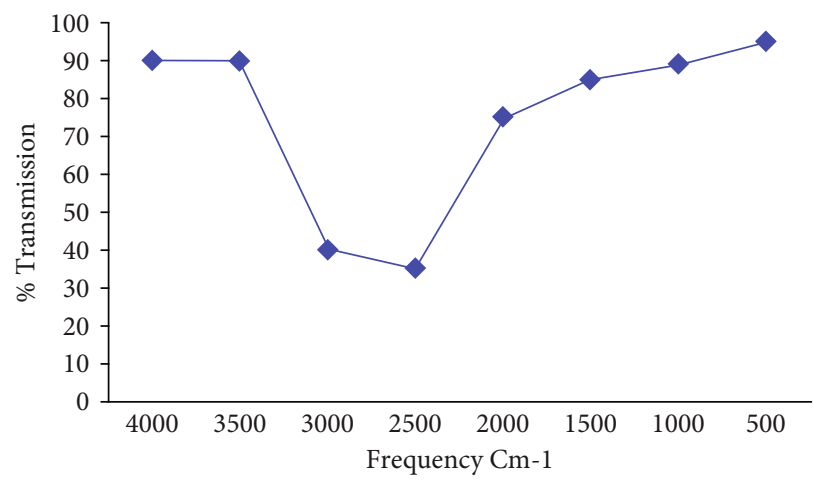

Figure 3: FTIR spectrum for polyethylene.

around $100 \mathrm{~m}$ for FTIR spectra. Figure 3 depicts the original polyethylene's infrared spectra. Figures 4-6 show the infrared spectra of various chlorinated polyethylene.

In the infrared range, chlorinated polyethylene's spectrum strongly matches with those polyvinyl chloride (1100 to 1300 per $\mathrm{cm}$ ). $\mathrm{C}_{2} \mathrm{H}_{4}$ is chlorinated in the creation of [-CHCl-], with only a few - $\mathrm{CCl}_{2}$ - units generated, according to the finding. The $\mathrm{CH}_{3}$ distortion phase can be seen at around $1380 \mathrm{~cm}^{-1}$ in the initial polyethylene spectrum. As the chlorine concentration of chlorinated polyethylene rises, this band becomes weak; yet, it exists in the spectra of objects carrying extra CL- than PVC, indicating that methyl groups are still available in the particles.

Figure 7 shows the comparative analysis of the classification accuracy for the existing and the proposed methods. It is evident from the graph that the proposed AI framework 


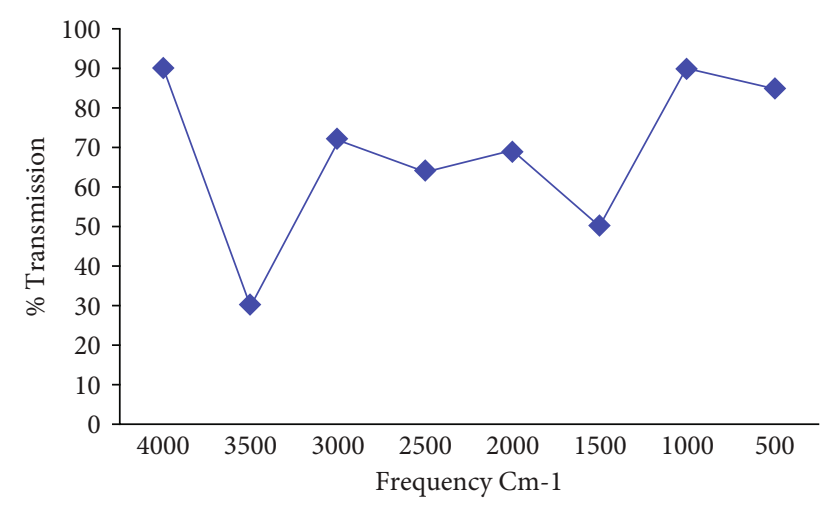

FIgURE 4: FTIR spectra for CPE-1.

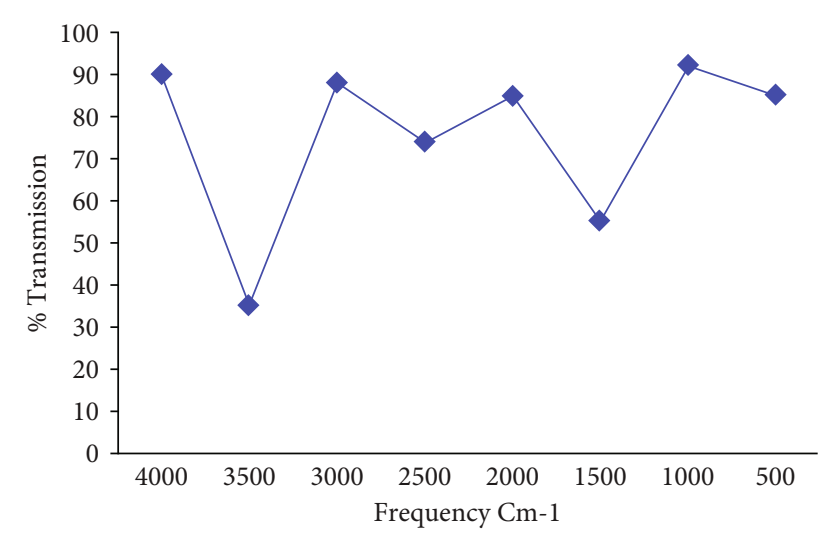

FIgURE 5: FTIR spectrum for CPE-2.



FIGURE 6: FTIR spectra for CPE-3.

outperforms the conventional approaches. The characterization of the properties of chlorinated polyethylene can be done better using the proposed classification algorithm when compared with the traditional classification approaches. This classification approach yielded accurate characterization results for the chlorinated polyethylene.

Table 4 shows the comparison of classification performance with respect to accuracy, precision, and recall.

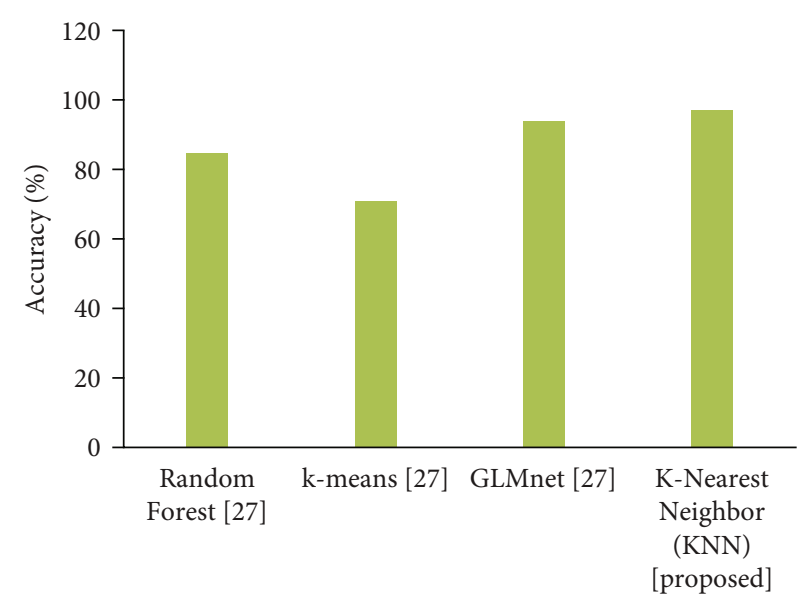

FIgURE 7: Comparison of classification accuracy for existing vs. proposed method.

TABLE 4: Comparison of classification performance.

\begin{tabular}{lcccc}
\hline S.no & Method used & $\begin{array}{c}\text { Accuracy } \\
(\%)\end{array}$ & $\begin{array}{c}\text { Precision } \\
(\%)\end{array}$ & $\begin{array}{c}\text { Recall } \\
(\%)\end{array}$ \\
\hline 1. & Random Forest [27] & 84 & 80 & 78 \\
2. & $k$-means [27] & 65 & 72 & 80 \\
3. & GLMnet [27] & 90 & 85 & 89 \\
4. & $K$-nearest neighbor & 97 & 92 & 91 \\
\hline
\end{tabular}

\section{Conclusion}

This article addresses the radical chlorination of $(\mathrm{C} 2 \mathrm{H} 4)_{n}$ in suspension below pressure and ultraviolet irradiation. The performance of characterization is improved by employing Bayesian optimization, an artificial intelligence method. Smaller particles had a lower weight and a higher chlorine concentration, according to the differential screening investigation. The FTIR spectra of CPE models demonstrate that the $\mathrm{CCl}$ stretching and twisting phases, also C-C shakings, produce large spikes as the reaction progresses. Because of the induction effect of $\mathrm{Cl}^{-}$particles contributed to the polymer network, the peak related to the $\mathrm{CH} 2$ - stretching mode grows wider.

\section{Data Availability}

The data used to support the findings of this study are available from the corresponding author upon request.

\section{Conflicts of Interest}

The authors declare that they have no conflicts of interest.

\section{References}

[1] A. Agrawal and A. Choudhary, "Deep materials informatics: applications of deep learning in materials science," Communications, vol. 9, no. 3, pp. 779-792, 2019. 
[2] K. Ahmed, "Performance evaluation of chlorinated polyethylene compatibilized-industrial waste-filled acrylonitrile butadiene rubber/low-density polyethylene blends," Journal of Vinyl and Additive Technology, vol. 22, no. 4, pp. 460-469, 2016.

[3] P. Charalampous, I. Kostavelis, T. Kontodina, and D. Tzovaras, "Learning-based error modeling in FDM 3D printing process," Rapid Prototyping Journal, vol. 27, no. 3, pp. 507-517, 2021.

[4] S. Jiang, "The effect of 4, 4'-methylene-bis-(2, 6-di-tert-butylphenol) on properties of polyvinyl alcohol/chlorinated polyethylene composites," Journal of Physics: Conference Series, vol. 1626, no. 1, p. 12182, 2020.

[5] B. Gui, X. Xu, S. Zhang, Y. Wang, C. Li, and D. Zhang, "Prediction of organic compounds adsorbed by polyethylene and chlorinated polyethylene microplastics in freshwater using QSAR," Environmental Research, vol. 197, p. 111001, 2021.

[6] A. Chaudhry and V. Mittal, "High-density polyethylene nanocomposites using masterbatches of chlorinated polyethylene/ graphene oxide," Engineering \& Science, vol. 53, no. 1, pp. 78-88, 2013.

[7] M. Hiles, Classifying Formulations and Tracking Accelerated Aging of Crosslinked Polyethylene Pipes by Applying Machine Learning Concepts to Infrared Spectra, Degree, Wiley, 2020.

[8] F. Cravero, S. A. Schustik, M. J. Martínez, G. E. Vázquez, M. F. Díaz, and I. Ponzoni, "Feature selection for polymer informatics: evaluating scalability and robustness of the FS4RVDD algorithm using synthetic polydisperse data sets," Journal of chemical information and modeling, vol. 60, no. 2, pp. 592603, 2020.

[9] Y. Zhao, Q. Li, X. Zhang, H. Li, J. Lu, and Z. Zhang, "High energy density and discharging efficiency achieved in chlorinated polyethylene films for high energy-storage applications," macromolecular Chemistry and Physics, vol. 219, no. 8, p. 1700621, 2018.

[10] S. Mondal, R. Ravindren, P. Bhawal, B. Shin, and S. Ganguly, "Combination effect of carbon nanofiber and ketjen carbon black hybrid nanofillers on mechanical, electrical, and electromagnetic interference shielding properties of chlorinated polyethylene nanocomposites," Composites Part B: Engineering, vol. 197, article 108071, 2020.

[11] Z. Li, Y. Zhangand, and H. Zhao, "Effect of electron beam irradiation on chlorinated polyethylene/vinyl acetate/nitrile rubber blend system," in IOP Conference Series: Materials Science and Engineering, p. 22110, 2020.

[12] A. P. Michel, A. E. Morrison, V. L. Preston, C. T. Marx, B. C. Colson, and H. K. White, "Rapid identification of marine plastic debris via spectroscopic techniques and machine learning classifiers," Environmental Science \& Technology, vol. 54, no. 17, pp. 10630-10637, 2020.

[13] D. Stefas, N. Gyftokostas, E. Bellou, and S. Couris, "Laserinduced breakdown spectroscopy assisted by machine learning for plastics/polymers identification," Atoms, vol. 7, no. 3, p. 79, 2019.

[14] A. B. Stanojevic, "Evaluation of life cycle in the process of production and management of PVC waste by LCA method and prediction of toxicity (TEQ) effect of dioxins using artificial neural network method and nonlinear regression," Annals of Environmental Science and Toxicology, vol. 5, 2021.
[15] L. Wang, L. Zhang, Y. Shi, and Z. Wang, "Thermoplastic elastomers based on ethylene-vinyl acetate copolymer and waste nitrile butadiene rubber powder blends compatibilized by chlorinated polyethylene," JOURNAL OF MACROMOLECULAR SCIENCE: PART B, vol. 57, no. 4, pp. 305-316, 2018.

[16] S. A. Osmani and F. Mahmud, "An integrated approach of machine algorithms with multi-objective optimization in performance analysis of event detection," Environment, Development and Sustainability, vol. 23, no. 2, pp. 1976-1993, 2021.

[17] P. Xu, T. Lu, L. Ju, L. Tian, M. Li, and W. Lu, "Machine learning aided design of polymer with targeted band gap based on DFT computation," The Journal of Physical Chemistry B, vol. 125, no. 2, pp. 601-611, 2021.

[18] W. Jiangand and N. Zhang, "Retracted article: research on characteristics of paper-plastic composite film based on intelligent optimization algorithm," Computing, vol. 25, no. S1, p. 27, 2021.

[19] Y. Li, L. Lv, W. Wang, J. Zhang, J. Lin, and J. Zhou, "Effects of chlorinated polyethylene and antimony trioxide on recycled polyvinyl chloride/acryl-butadiene-styrene blends: flame retardancy and mechanical properties," Polymer, vol. 190, article $122198,2020$.

[20] Y. Wang, T. Xie, A. France-Lanord, A. Berkley, J. A. Johnson, and Y. Shao-Horn, "Toward designing highly conductive polymer electrolytes by machine learning assisted coarse-grained molecular dynamics," chemistry of Materials, vol. 32, no. 10, pp. 4144-4151, 2020.

[21] S. P. Ong, "Accelerating materials science with highthroughput computations and machine learning," computational Materials Science, vol. 161, pp. 143-150, 2019.

[22] H. Yamada, C. Liu, S. Wu, Y. Koyama, S. Ju, and J. Shiomi, "Predicting materials properties with little data using shotgun transfer learning," ACS central science, vol. 5, no. 10, pp. 17171730, 2019.

[23] E. Kim, Z. Jensen, A. van Grootel, K. Huang, M. Staib, and S. Mysore, "Inorganic materials synthesis planning with literature-trained neural networks," Journal of chemical information and modeling, vol. 60, no. 3, pp. 1194-1201, 2020.

[24] L. E. Vivanco-Benavides, C. L. Martínez-González, C. Mercado-Zúñiga, and C. Torres-Torres, "Machine learning and materials informatics approaches in the analysis of physical properties of carbon nanotubes: a review," Computational Materials Science, vol. 201, article 110939, 2022.

[25] X. G. Zhao, K. Zhou, B. Xing, R. Zhao, S. Luo, and T. Li, "JAMIP: an artificial-intelligence aided data-driven infrastructure for computational materials informatics," Science Bulletin, vol. 66, no. 19, pp. 1973-1985, 2021.

[26] T. Ünügüland and B. Karaağaç, "Vulcanization of chlorinated polyethylene/chloroprene rubber compounds at lower temperatures in the presence of reactive silanes," Journal of applied polymer Science, vol. 138, no. 23, p. 50544, 2021.

[27] M. Doaei, M. S. Tavallali, and H. Nejati, "Fault classification in electrofusion polyethylene joints by combined machine learning, thermal pulsing and IR thermography methods - a comparative study," Infrared Physics \& Technology, vol. 96, pp. 262-266, 2019. 